\title{
Analysis of ECG in Athletes Running in Mountain Route Conditions
}

\author{
Pedro Gomis ${ }^{1,2}$, Eduard Guasch $^{3}$, Alexandre Perera $^{1,2}$, Lluis Mont $^{3}$, Pere Caminal ${ }^{1,2}$ \\ ${ }^{1}$ ESAII Dept, EEBE, CREB, Universitat Politècnica de Catalunya, Barcelona, Spain \\ ${ }^{2}$ CIBER - BBN (Bioenginering, Biomedicine and Nanomedicine), Spain \\ ${ }^{3}$ Arrhythmia Section, Hospital Clínic, CIBERCV, IDIBAPS, Universitat de Barcelona, Spain
}

\begin{abstract}
The purpose of this work is to analyse changes in ECG and heart rate variability (HRV) metrics in athletes during $10 \mathrm{~km}$ running in mountain route conditions.

Eighteen healthy athletes carrying a 12-lead ECG GE Holter recorder and a heart rate monitor ran a route with slopes similar as trail races. QRS duration, QRS area, $R$ wave peak, ST elevation at J-point and J+60 ms, ST slope and $T$-wave peak indices were computed after signalaveraging ECG segments at different sloping stages of running (S1-S6) and at a control stage (SO) before running. HRV analysis included standard time and frequency metrics: mean RR (normal, $N$ ) interval, SDNN, RMSSD, low and high frequency absolute and normalized power ( $L F, H F, L F n, H F n)$ and $L F / H F$ ratio.

$Q R S$ area and R-peak were reduced during stage 1 in lateral leads, comparing to rest before running. ST slope was significantly higher during upslope stages in leads II, $V 4$ and V5. T-wave amplitude increased significantly in precordial leads during upload running. ST segment depressed in leads II, III and V5 respect control. Mean $R R, S D N N, L F$ and LFn showed high significant differences ( $p<0.01)$ among stages and $H F$ and $L F / H F$ were also varying $(p<0.05)$. Changes of ECG and HRV indices can help understanding the cardiac function in runners performing extreme stress.
\end{abstract}

\section{Introduction}

Electrocardiographic analysis in endurance athletes is currently a major challenge to correctly differentiate changes in ECG indices associated with severe cardiovascular problems from favourable physiological adaptations due to intense exercise that causes the athlete's heart. Physical exercise has widely been promoted for heart disease rehabilitation and prevention. However, sudden cardiac death (SCD) is one of the main causes of mortality of athletes during sport [1]. Moreover, performing intensive exercise has been linked by several studies with atrial fibrillation [2,3], ventricular arrhyth- mias and ischemic heart disease [4]. Physiological cardiac remodelling due to intense exercise produces changes in the morphology and associated ECG indices.

A study of elite athletes during their active and retired years showed that one third of them presented benign early repolarization patterns (upsloping ST-segment), which remained in half of the cases after retirement and no episodes of SCD was found after a long follow-up period [5]. Hence, an international committee of experts in sports cardiology and medicine has proposed some standards for ECG interpretation in athletes to distinguish normal ECG alterations in sportsmen from abnormal ECG findings [6]. For example, among normal ECG morphologies in athlete, that can be considered physiological cardiac remodelling, are: sinus bradycardia $(\geq 30 \mathrm{bpm}), 1^{\circ}$ atrioventricular block (PR interval $>200$ $\mathrm{ms}$ ) or early repolarization patterns.

In sports medicine, heart rate variability (HRV) has also been considered an important tool to assess the autonomic nervous system response to different physical efforts. Decreases and increases in vagal-derived indices of HRV have been suggested to indicate negative and positive adaptations, respectively, to endurance training schedules [7].

Since most ECG measurements during exercise are obtained under controlled lab conditions, this work aims to analyse variations in ECG parameters and HRV metrics in athletes during long-distance running in mountain route conditions.

\section{Material and methods}

\subsection{Protocol setup and data acquisition}

A group of 22 enlisted volunteers who perform regular exercise, aged 21-28 years, gave their written consent to participate in this study. ECG Holter records from four subjects were not included because low signal quality. Therefore, analysis was performed on 18 participants ( 9 males and 9 female); their mean \pm SD of age, height and weight were $23 \pm 2 \mathrm{yr}, 173 \pm 11 \mathrm{~cm}$ and $65.6 \pm 9.8 \mathrm{~kg}$, respectively. Participants reported their time spent in 

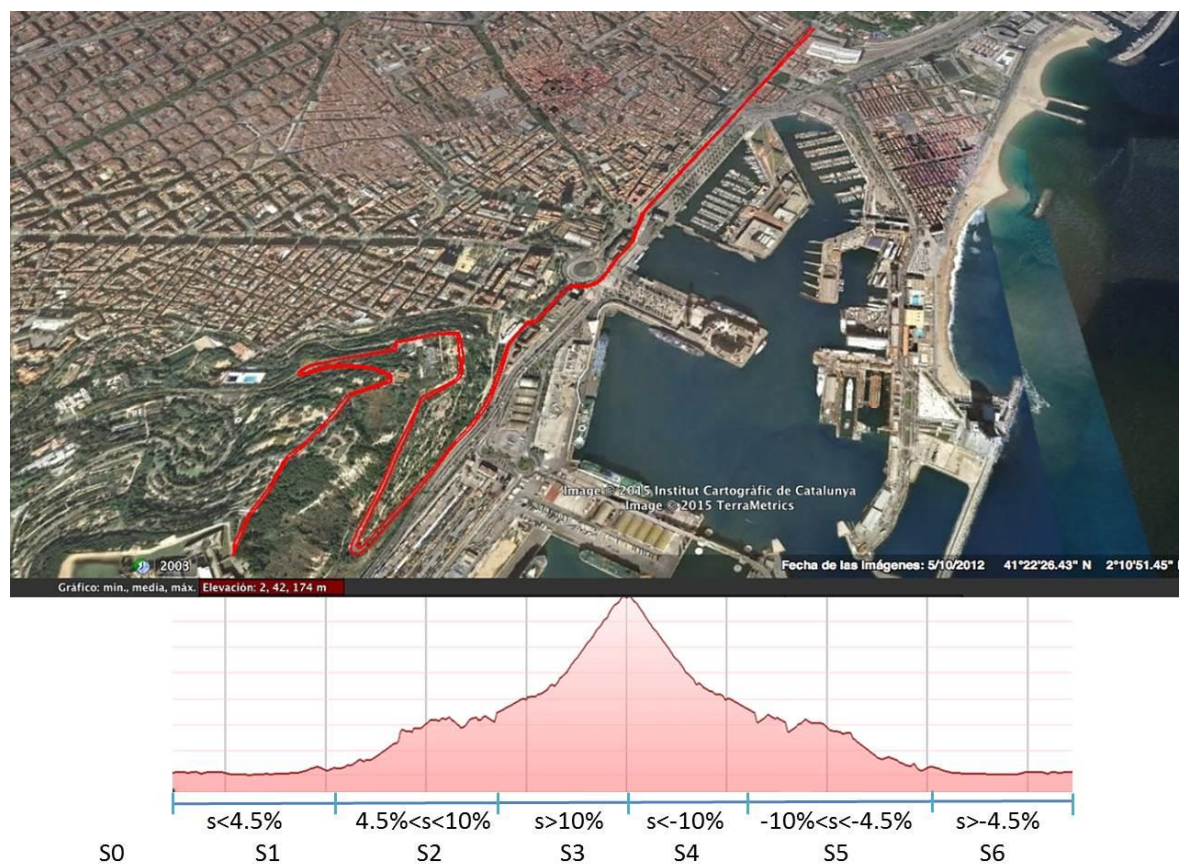

Figure 1. Route map and elevation profile. Stages S0 (rest) and S1-S2 (running); s: slope.

exercising in a range of 3 to $12 \mathrm{~h} /$ week $(6.4 \pm 4.1 \mathrm{~h} /$ week $)$, considered as moderate-intensity physical activity.

To analyse the changes in ECG morphology and HRV metrics, a running route was designed to include a range of slopes in Montjuich Mountain, Barcelona, as in trail or ultra-trail races and to be long enough to include a range of situations that can be found in open field running. Fig. 1 shows the route map and the elevation profile. The total distance of the running route was $9.87 \mathrm{~km}$. Minimum and maximum elevations were $2 \mathrm{~m}$ and $174 \mathrm{~m}$, respectively.

ECG data were obtained with two devices. A 12-lead GE SEER 12 Holter (GE Healthcare Getemed AG, Teltow, Germany) recorded 12-lead ECG at a sampling frequency of $128 \mathrm{~Hz}$ and a Polar V800 heart rate monitor (HRM) with a Polar H7 Heart Rate Sensor chest strap (henceforward, V800) simultaneously recorded the RR intervals at a sampling frequency of $1000 \mathrm{~Hz}$. Electrodes were placed in the standard configuration for a 12-lead ECG and elastic electrode V800 HRM belt was placed in accordance with the manufacturer's guidelines. The position of the runner was recorded by the V800, with a GPS sampling frequency of $1 \mathrm{~Hz}$.

ECG indices and HRV metrics were obtained in 5-min segments during seven stages for each runner. The stages during the running route were: S1, during the first part of the course with a running slope lower than $4.5 \%$; S2, with a positive running slope between $4.5 \%$ and $10 \%$; S3, before reaching the top of the mountain, with an average positive running slope of $12 \%$; S4, after the mountain's top, with an average negative running slope of $-12 \%$; S5, with a negative running slope between $-10 \%$ and $-4.5 \%$; S6, during the last stage of the course with running slope lower than $4.5 \%$ (Fig. 1). Additionally, a control resting stage (S0) before running was included. Stages selections was based on position of runners during the route using Polar V800 GPS signal, which was corrected using the GPS Visualizer, a utility that creates maps and profiles from geographic data. Data was taken from the NASA database of Shuttle Radar Topography Mission (SRTM).

\subsection{Preprocessing}

The GE Holter ECG recordings were uploaded with GE MARS 8.0.2 software and raw signals and beat or event locations, with their classification annotation, were exported in MIT format for posteriorly been read, using WFDB Toolbox for MATLAB and Octave from Physionet $[8,9]$. The ECG signals were resampled from $128 \mathrm{~Hz}$ to $1000 \mathrm{~Hz}$ through spline interpolation, giving a temporal resolution of $1 \mathrm{~ms}$. R-wave peak time positions were automatically detected in the ECG signals using a custom peak detection algorithm. ECG recordings and accuracy of R-wave detection were verified by visual assessment of the data. Normal-to-normal (NN) R-R intervals were obtained. In order to assess the HRM performance, alignment of the two R-R data series (from the V800 and the Holter) was done using minimum distance criteria.

Continuous ECG recordings were signal averaged to attain low noise level in analysing ECG morphology. For each 5-min segment of the stages, an averaged beat for each lead was obtained using conventional ensemble averaging of the normal sinus beats, with a custom signal averaging algorithm. 


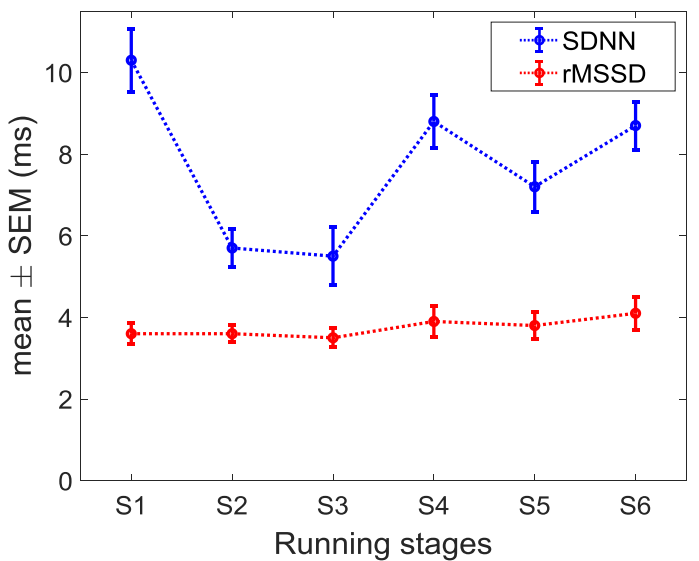

Figure 2. SDNN and rMSSD mean \pm SEM during running.

\subsection{HRV and ECG analysis}

As HRV time domain indices, heart rate (HR) mean, the standard deviation of normal R-R intervals (SDNN) and the square root of the mean square differences of successive R-R intervals (rMSSD) were calculated.

HRV frequency domain indices were obtained using the power spectral density of the processed R-R intervals using techniques previously described [10]. These indices were: low frequency power (LF: $0.04-0.15 \mathrm{~Hz}$ ), high frequency power (HF: $0.15-0.4 \mathrm{~Hz}$ ), LF/HF ratio, normalized LF (LFnorm) and normalized HF (HFnorm).

Averaged ECG beats were automatically delineated to obtain QRS onset and offset using a multi-lead approach [11]. Depolarization changes during the running stages were measured with the R-wave amplitude peak, the QRS duration and QRS area indices. While repolarization variations were calculated with the ST-segment elevation at J-point and at J+60 ms, the ST-segment slope and the $\mathrm{T}$-wave amplitude peak. $\mathrm{R}$ and $\mathrm{T}$ wave amplitudes and ST segment deviations were computed for each single lead using the PR segment as the isoelectric level. QRS duration was globally calculated for each beat.

$\mathrm{R}-\mathrm{R}$ intervals and HRV indices obtained from the Holter ECG and V800 were compared in order to assess the reliability of using HRV indices from heart rate monitors to assess autonomic response. V800 R-R intervals were corrected by means of a custom algorithm based on previous correction algorithms used at rest [12].

\section{Results and discussion}

As expected, HR significantly increased from resting, $87.2 \pm 6.3 \mathrm{bpm}$, to a range of $160.0 \pm 8.7$ to $183 \pm 8.9 \mathrm{bpm}$ during the 6 evaluated stages of running (S1-S6). Moreover, the steepest S3 produced faster HR respect to each of the running stages $(\mathrm{p}<0.05)$. Fig. 2 shows significant changes of SDNN, as the overall HRV index, during the running stages. SDNN was reduced at S3 respect $\mathrm{S} 1$ and downsloping stages $(\mathrm{p}<0.05)$. No changes were observed in rMSSD index.

LF significantly decreased from S1 to the steepest S3 and recovered to former values during final stages $(\mathrm{p}<0.05)$. Normalized LF and HF and LF/HF balance indices during running are observed in Fig. 3. LFnorm was considerably reduced during the effort of S3 compared with S1 and S4 to S6 ( $p<0.001)$. LF/HF ratio also decreased during $\mathrm{S} 3$ with respect to $\mathrm{S} 1$ and downsloping stages $(\mathrm{p}<0.01)$. HFnorm increased during $\mathrm{S} 3$ compared to the first stage and reduced during the first downslope stage $\mathrm{S} 4(\mathrm{p}<0.05)$.

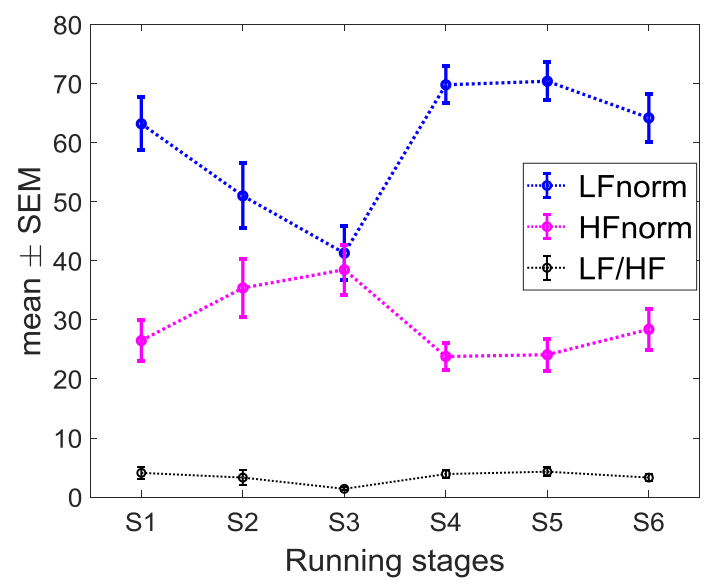

Figure 3 LFnorm, HFnom and LF/HF during the stages.

During the 6 running stages and for all participants a total of 91,825 R-R intervals were detected by the V800 HRM; no systematic differences were found between the V800 and the Holter. The total error rate was $0.71 \%$, being the loss of 1 or several R-R intervals the most frequent error. Correlation between corrected V800 R-R intervals and the Holter were $0.99(\mathrm{p}<0.01)$. Limit of agreement, using Bland Altman analysis with multiple and non-constant observations per individual, were -3.61 to 3.63. The bias obtained when comparing Holter and corrected V800 R-R intervals was less than $1 \mathrm{~ms}$.

No significant differences were found for time domain and frequency domain indices computed from the corrected V800 and Holter signals, except for the power in the low frequency band $(\mathrm{p}<0.05)$.

ECG morphology indices from the averaged signals of every 5-min segment stages showed changes on several leads of different parameters. QRS duration maintained similar values during all the analysed stages. Fig. 4 shows a markedly reduction of QRS area from rest to $\mathrm{S} 1$ stage in inferior leads II and III ( $<<0.001)$. R-wave amplitude decreased from S0 to S1 stage in lead II $1.9 \pm 0.4$ vs. $1.6 \pm 0.4 \mathrm{mV}(\mathrm{p}<0.05)$.

The ST-segment at the J-point significantly depressed from rest to $\mathrm{S} 1$ in lateral and inferior leads $(\mathrm{p}<0.001)$, as 
depicted in Fig 5 (A). ST-segment at J+60ms depressed in leads II and III from S0 to S1 ( $\mathrm{p}<0.001)$. The ST-segment slope (Fig. 5(B)), measured as the difference between STsegment at $\mathrm{J}+60 \mathrm{~ms}$ and at $\mathrm{J}$-point, was upward sloping during all running stages in leads II, V4 and V5, while maintained horizontal during rest (S0). T-wave amplitude increased significantly in precordial leads (V3-V5) during upload running stages with respect to $S 0(p<0.05)$.

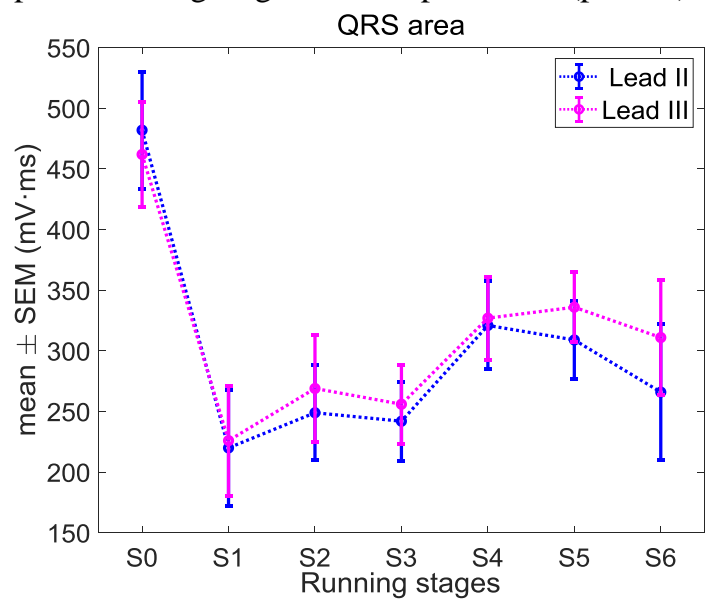

Figure 4. QRS area changes during running.
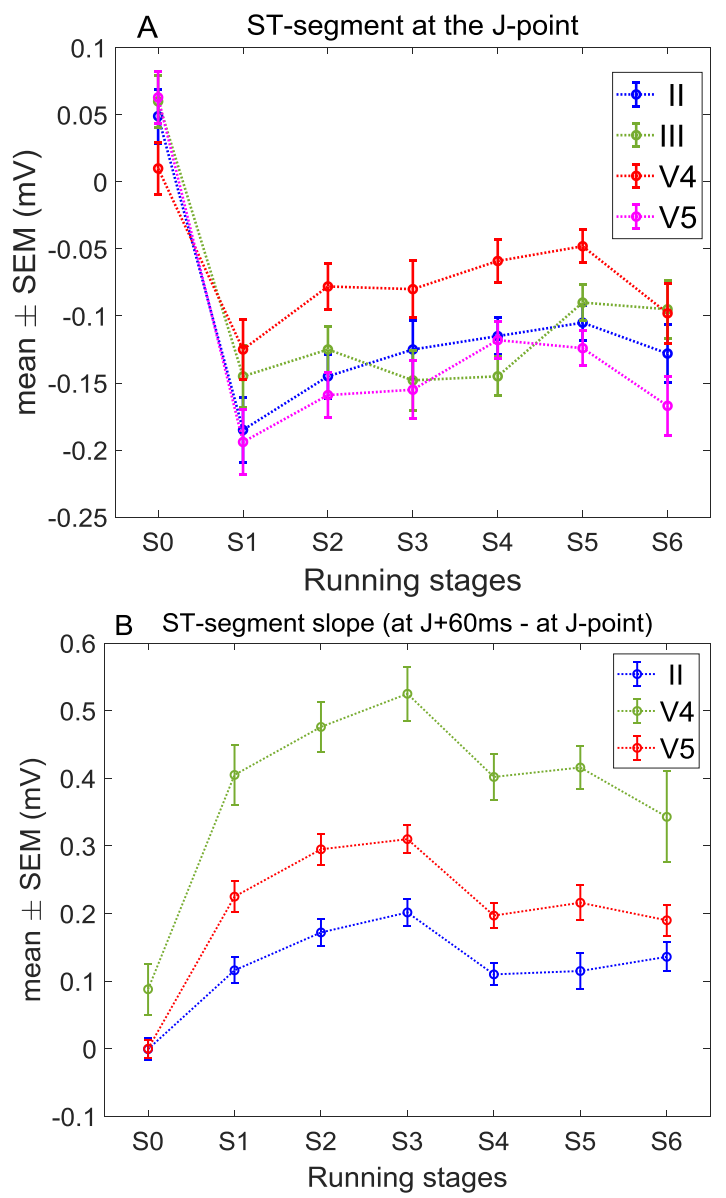

Figure 5. ST-segment deviation at J-point (A) and slope (B) during the running stages.

\section{Conclusion}

In sports medicine it is important to evaluate the normal cardiac adaptation to the continued effort to differentiate it from possible cardiovascular risk anomalies. Changes in the ECG and HRV indices while running mountain routes can help to better understand cardiac function in runners who perform extreme stress.

\section{Acknowledgements}

This work was supported by TEC2014-60337-R and DPI2017-89827-R grants from the Government of Spain.

\section{References}

[1] Harmon KG, Asif IM, Klossner D, et al. Incidence of sudden cardiac death in national collegiate athletic association athletes. Circulation 2011;123:1594-600.

[2] Mont L, Sambola A, Brugada J, et al. Long-lasting sport practice and lone atrial fibrillation. Eur Heart J 2002; 23:477-82.

[3] Morseth B, Graff-Iversen S, Jacobsen BK et al. Physical activity, resting heart rate, and atrial fibrillation: the Tromsø Study. Eur Heart J 2016; 37(29):2307-2313.

[4] Guasch, E, Nattel, S. CrossTalk proposal: Prolonged intense exercise training does lead to myocardial damage. J Physiology. 2013;591(20), 4939-4941.

[5] Serra-Grima R, Doñate M, Álvarez-García et al. Long-term follow-up of early repolarization pattern in elite athletes. Am J Med 2015;128:192.e1-e9.

[6] Drezner JA, Ackerman MJ, Anderson J, Ashley E et al. Electrocardiographic interpretation in athletes: the 'Seattle criteria'. Br J Sports Med 2013; 47(3):122-124.

[7] Plews D, Laursen P, Stanley J, Kilding A, Buchheit M. Training Adaptation and Heart Rate Variability in Elite Endurance Athletes: Opening the Door to Effective Monitoring. Sports Med 2013;43(9):773-781.

[8] Silva I, Moody G. An Open-source Toolbox for Analysing and Processing PhysioNet Databases in MATLAB and Octave. Journal of Open Research Software 2014;2(1):e27.

[9] Goldberger AL, Amaral LAN, Glass L, et al.. PhysioBank, PhysioToolkit, and PhysioNet: Components of a New Research Resource for Complex Physiologic Signals. Circulation 2000;101(23):e215-e220.

[10] Gomis P, Álvarez J, Caminal P, Cinca J. Analysis of heart rate variability indices after selective acute atrial ischemia in humans. Computing in Cardiology 2017;44:149-1-4.

[11] Pahlm O, Sörnmo L. Data processing of exercise ECG's. IEEE Trans Biomed Eng 1987;34:158-65.

[12] Giles D, Draper N, Neil W. Validity of the Polar V800 heart rate monitor to measure RR intervals at rest. Eur J Appl Physiol 2016;116:563-571.

Address for correspondence.

Pedro Gomis.

Universitat Politècnica de Catalunya,

Av Diagonal, 647, ETSEIB, CREB, 08028 Barcelona, Spain. pedro.gomis@upc.edu. 